\title{
Das Gesundheitswesen zwischen Seuchenpolizei und Public Health
}

\section{Christiane Meier}

Dr. med., Vorstandsmitglied der Schweizerischen Gesellschaft der Fachärztinnen und -ärzte für Prävention und Gesundheitswesen (SGPG)

Um das schweizerische Gesundheitswesen zu verstehen, lohnt es sich, seine Entwicklung zu studieren. Das nachfolgend besprochene Buch «Von der Seuchenpolizei zu Public Health» zeigt diese Geschichte auf und stellt den Kontext zu den jeweiligen gesellschaftspolitischen Entwicklungen her.

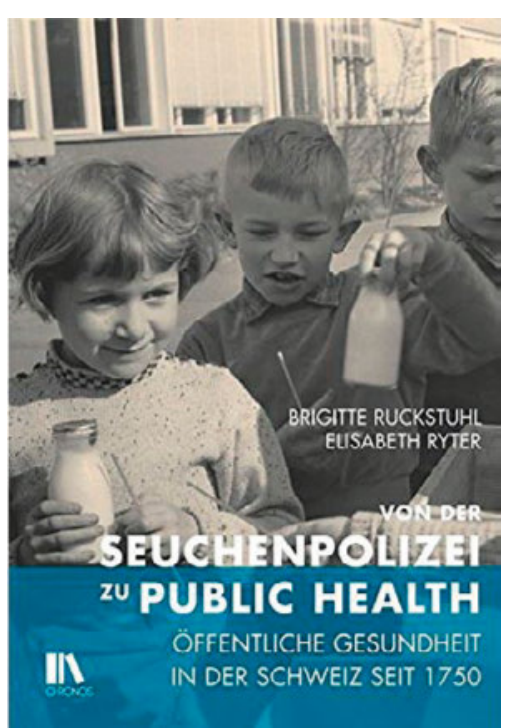

Von der Seuchenpolizei zu Public Health: Öffentliche Gesundheit in der Schweiz seit 1750 Brigitte Ruckstuhl, Elisabeth Ryter

Zürich: Chronos; 2017.

ISBN: 978-3-0340-1388-8

344 Seiten. 42 CHF.

Im neu erschienenen Buch «Von der Seuchenpolizei zu Public Health» beschreiben Brigitte Ruckstuhl und Elisabeth Ryter die Geschichte des Gesundheitswesens der Schweiz. Sie tun dies anschaulich illustriert mit zeitgenössischen Bildern, Portraits und Dokumenten. Besonders bereichernd sind zudem die wortgetreuen Äusserungen in Originalsprache von prägenden Persönlichkeiten.

Auch die eiligen Leserinnen und Leser finden raschen Zugang zu den historischen Ursprüngen des heute so komplexen wie kontrovers diskutierten Schweizer
Gesundheitswesens, denn die Einleitung zum Buch sowie die Einführung und das Fazit zu jedem Kapitel bieten einen guten und raschen Überblick.

\section{Hygiene und Gesundheit}

Das Buch setzt um 1750 ein, als mangelnde Hygiene und grassierende Infektionskrankheiten die Organisation eines Gesundheitswesens nötig machten. Dass die Gesundheit des Individuums von seinen (hygienischen) Lebensumständen geprägt ist, war damals

\section{Auch die eiligen Leserinnen und Leser finden raschen Zugang zu den historischen Ursprüngen des Schweizer Gesundheitswesens.}

selbstverständlicher als in der heutigen Zeit, in der das Buch endet und mit einer Diskussion über die Zukunft der personalisierten oder individualisierten Medizin einen spannenden Bogen schlägt. Die Autorinnen zeigen auf, wie mit der «Medizinierung» soziale Aspekte in den Hinter- und der individualisierte Blick auf die Gesundheit in den Vordergrund traten. So wurde die "öffentliche Betrachtung der Gesundheit» anfangs des 20. Jahrhunderts zwar zu einem eigenständigen Handlungsfeld erklärt, verlor gegenüber der biomedizinischen Sichtweise jedoch zunehmend an Bedeutung.

Auf dieser Zeitreise wird eindrücklich dargestellt, wie gesundheitspolitische Entwicklungen häufig aktuellen gesellschaftspolitischen Problemen und Debatten folgen und wie einzelne Persönlichkeiten entscheidende Entwicklungen anschieben können. Man erfährt von den Lebensumständen der Bevölkerung in verschiedenen Epochen, von den vorherrschenden gesundheitlichen Problemen und von Lösungsansätzen. Dabei wird 
die Schweizer Geschichte nicht isoliert, sondern im Kontext ausländischer Entwicklungen beleuchtet. Nachgezeichnet werden etwa die Einflüsse internationaler Hygienekongresse ab 1850 oder der OttawaCharta von 1986 und man erfährt, welche Pioniere ab 1956 in den USA einen Master in Public Health absolvierten, lange bevor dies in der Schweiz möglich war.

\section{Der schulärztliche Dienst}

Anschaulich skizziert wird auch die Geschichte des schulärztlichen Dienstes, der sich nach Einführung des Schulobligatoriums 1874 hauptsächlich mit Haltungsschäden und den richtigen Massen von Schulbänken befasste - das Turnobligatorium für Mädchen

\section{Die Schweizer Geschichte wird nicht isoliert, sondern im Kontext ausländischer Entwicklun- gen beleuchtet.}

wurde aber gleichwohl erst 1972 bundesgesetzlich verankert. Es finden sich auch Parallelen zu heute: Den Schulärzten war eine Behandlung kranker und psychisch angeschlagener Kinder untersagt - die freie Arztwahl der Eltern musste garantiert bleiben! Und bezüglich Impfungen lässt sich ein Arzt 1880 mit den Worten zitieren: «... von Oben her Order, von Unten her Renitenz. In der Mitte steht der Impfarzt mit seinem Verdruss ...»

Etwas mehr Detailreichtum würde man sich als Lesende für die Zeit während und zwischen den Weltkriegen wünschen. Hier bleibt das Buch dürftig. Vielleicht weil keine Public-Health-relevanten Impulse aus dem Ausland zu erkennen sind und die Schweiz von der Kriegsmedizin verschont blieb?

\section{Drogen, HIV und Aids}

Ausführlicher gehen die Autorinnen dann wieder der Drogenproblematik und deren gesundheitlichen sowie politischen Auswirkungen nach. Auch beim damaligen Umgang mit HIV/Aids und dessen Einflüssen gelingt es ihnen, die gesamtgesellschaftlichen Zusammenhänge aufzuzeigen. Sie machen verständlich, welche Massnahmen in welcher Zeit möglich waren, welche Umstände es erlaubten, auf die Ausbreitung von AIDS mit einer bis dahin beispiellosen Kampagne zu reagieren. Chronische Krankheiten, die heute als ein Hauptgrund für die Kostenexplosion - übrigens schon 1970 als «Rakete mit ungewisser Landung» bezeichnet - angeführt werden, sind ungleich schwieriger anzugehen. Mit der vom Bund erarbeiteten NCD-Strategie - die es gerade noch knapp ins Buch geschafft hat - rücken die Einflüsse der Lebensumstände und sozialer Determinanten auf die Gesundheit wieder vermehrt in den Fokus. Auch wenn die Kausalitäten aktueller nicht übertragbarer Krankheiten schwieriger zu erklären sind, möchte man am Ende der Lektüre nochmals zurückblättern zu den Anfängen, als es den

\section{Etwas mehr Detailreichtum würde man sich} als Lesende für die Zeit während und zwischen den Weltkriegen wünschen.

Gelehrten gelungen ist, den Gesundheitszustand als Resultat des eigenen Lebenswandels zu erklären.

\section{Mein Fazit}

«Von der Seuchenpolizei zu Public Health» ist eine gesunde Mischung aus einem informativen Sachbuch und einer Abendlektüre zum Geniessen! 